\title{
Retos y perspectivas en el ecosistema de la información biomédica
}

\section{Challenges and perspectives in the biomedical information ecosystem}

\author{
Gonzalo Casino
}

Cómo citar este artículo:

Casino, Gonzalo (2019). "Retos y perspectivas en el ecosistema de la información biomédica”. El profesional de la información, v. 28, n. 2, e280205.

https://doi.org/10.3145/epi.2019.mar.05

Artículo recibido el 11-03-2019

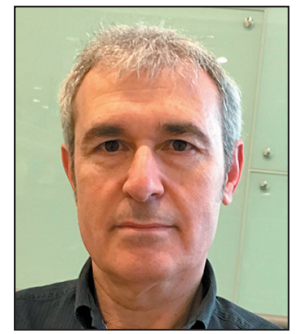

\author{
Gonzalo Casino \\ http://orcid.org/0000-0003-1872-4130
}

Observatorio de la Comunicación Científica, Departamento de Comunicación, Universitat Pompeu Fabra, Barcelona, España gonzalo.casino@upf.edu

\section{Resumen}

Los nuevos flujos informativos y el derrumbe del monopolio periodístico en la intermediación con el público han creado oportunidades y disfunciones en la información biomédica. A los problemas de la exageración y la comercialización, se han añadido los relacionados con la falta de filtros y la difusión de la mala ciencia. En este artículo se discuten los retos que plantean estos problemas y las perspectivas en la investigación académica, la comunicación y el periodismo.

\section{Palabras clave}

Comunicación biomédica; Periodismo científico; Divulgación; Noticias médicas; Noticias sobre salud; Información de salud; Impacto mediático; Calidad de la información; Desinformación; Mala ciencia.

\begin{abstract}
The new information flows and the collapse of the journalistic monopoly in the intermediation with the public, have created opportunities and dysfunctions in biomedical information. To the problems of hype and commercialization, those related to the lack of filters and the diffusion of bad science have been added. This article discusses the challenges posed by these problems and the perspectives in academic research, communication and journalism.
\end{abstract}

\section{Keywords}

Biomedical communication; Scientific journalism; Popularization; Medical news; Health news; Health information; Media impact; Quality of the information; Disinformation; Bad science.

\section{Introducción}

La información sobre biomedicina se ha desarrollado como una rama central y vigorosa del árbol de la ciencia y la información científica. En los tres ámbitos principales de análisis, la investigación académica, la comunicación y el periodismo, los temas relacionados con la medicina y la salud ocupan un espacio relevante y pujante en el contexto de las ciencias. Aunque la medicina no es propiamente una ciencia, sino más bien una ciencia aplicada o una práctica profesional basada de forma prioritaria pero no exclusiva en el conocimiento científico, se ha erigido en el imaginario popular como la ciencia por excelencia, por delante de la física y la química, al tiempo que la población reconoce un mayor interés por los temas de medicina y salud que por los de ciencia y tecnología (Bauer, 1998; Fecyt, 2018). 
La biomedicina no tiene una definición y unos límites precisos. Incluye las disciplinas médicas (clínicas, sociales y humanísticas), las ciencias de la vida que les dan soporte, y otras disciplinas con el prefijo bio relacionadas con la medicina y la salud. Algunas estimaciones indican que la biomedicina representa casi la mitad del conjunto de la producción científica (Camí; Méndez-Vásquez; Suñén-Pinyol, 2008). En la visualización de la estructura de la ciencia que ofrece la web SCImago (figura 1), la biomedicina ocupa unas dos quintas partes, una proporción similar a la que representan los 1.200.000 ar-

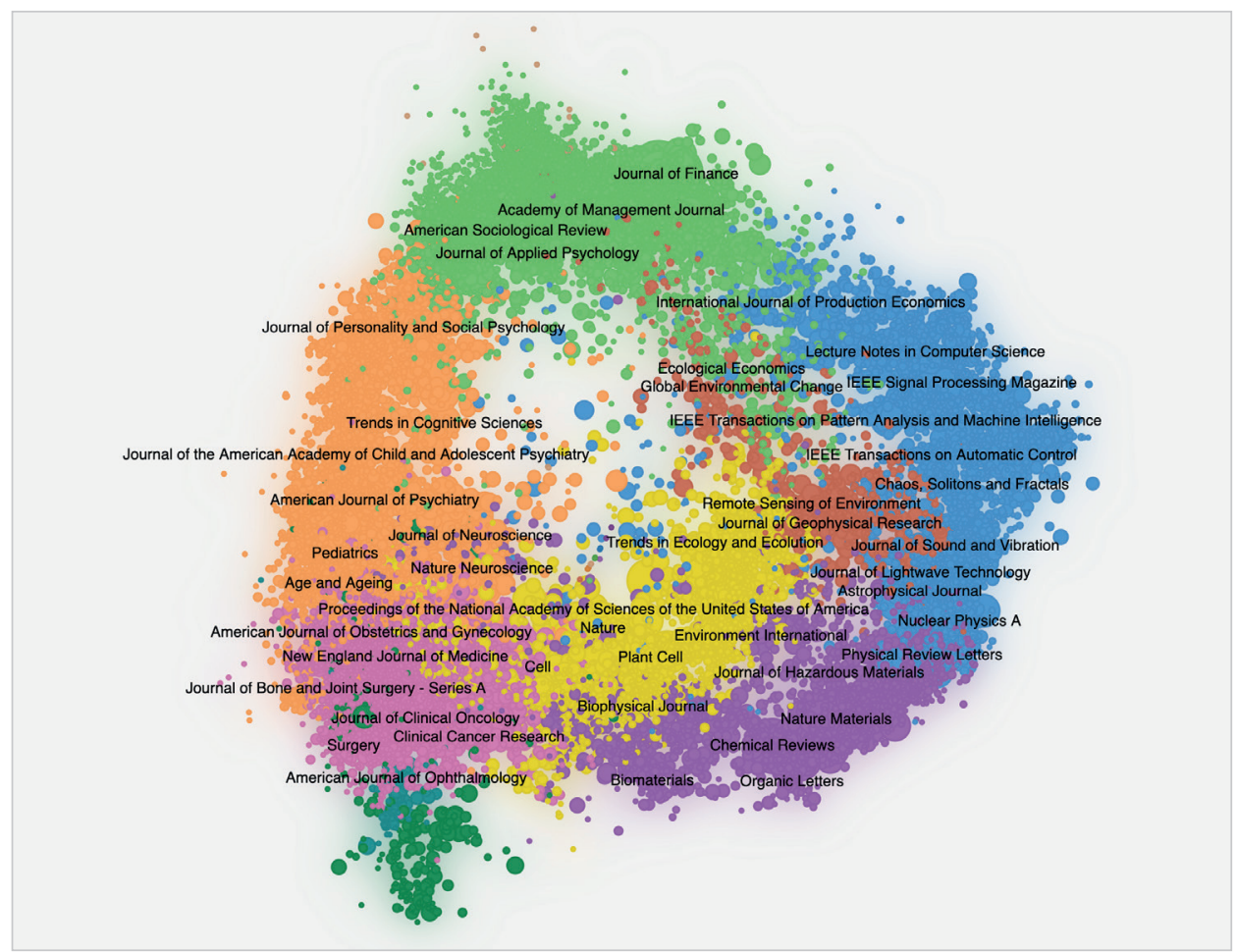

Figura 1. Representación de la estructura de la ciencia de SCImago basada en la base de datos Scopus https://www.scimagojr.com/shapeofscience tículos anuales recogidos en la base de datos Medline frente a los casi tres millones que indexa Scopus también cada año. En muchos medios de comunicación, la información sobre biomedicina representa tanto o más que la dedicada al resto de la ciencia.

La irrupción de internet y las redes sociales ha alterado los flujos informativos y el papel tradicional de los distintos agentes que participan en la información biomédica (científicos, revistas académicas, centros de investigación, periodistas y medios de comunicación, divulgadores, comunicadores, etc.), apareciendo nuevas oportunidades de comunicación a la vez que nuevos problemas. En este artículo se analizan los principales problemas, retos y perspectivas en la investigación, la comunicación y el periodismo sobre biomedicina, unas veces de forma conjunta y otras por separado, con el objetivo de ofrecer una visión integrada y a la vez desglosada por agentes y actividades.

\section{Ecosistema de la información biomédica}

El análisis del ecosistema de la información biomédica implica el uso preciso de algunos términos recurrentes, en concreto los de información, comunicación, divulgación y periodismo. A efectos de reducir la ambigüedad, y sin ánimo de hacer definiciones exhaustivas, se aclara de entrada el sentido en el que se usan aquí estos términos:

\subsection{Información, comunicación, periodismo y divulgación}

En general, cuando hablamos de información nos referimos a los mensajes y cuando hablamos de comunicación nos referimos al intercambio de mensajes (figura 2). En la comunicación biomédica, interesa distinguir dos variedades especializadas: la comunicación profesional y el periodismo profesional. La primera es la que realizan los gabinetes de comunicación y comunicadores profesionales que difunden notas de prensa y otros mensajes de interés para la institución para la que trabajan; se trata, por tanto, de una comunicación "interesada". La segunda es la que realizan los periodistas profesionales que trabajan para algún medio de comunicación y elaboran mensajes de interés

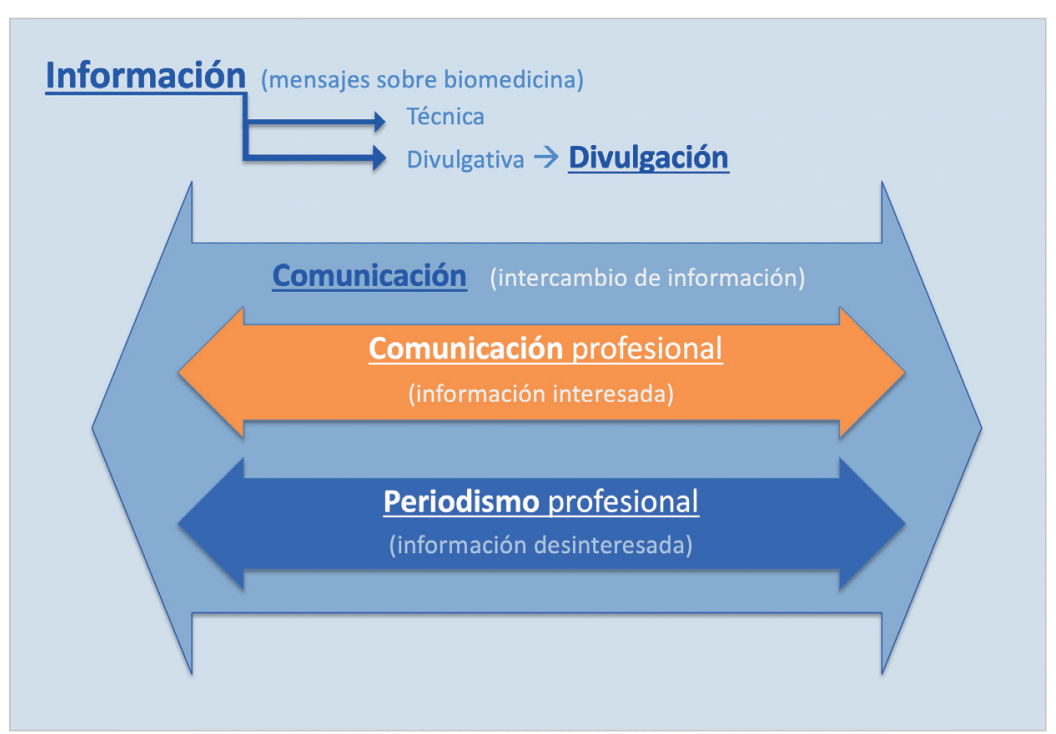

Figura 2. Información, comunicación, periodismo y divulgación 
público con los métodos, normas y códigos propios de la profesión periodística; se trata, por tanto, de una comunicación "desinteresada" o de "interés general".

En cuanto al término divulgación, debe entenderse como una característica que posee, en mayor o menor medida, la información biomédica (existe un lenguaje divulgativo en oposición al técnico) a la vez que una actividad que desarroIlan en grado variable los agentes (periodistas, comunicadores profesionales y científicos divulgadores) y que puede implicar, en algunos casos, una profesionalización (por ejemplo, los divulgadores de los museos de ciencia y los autores de obras divulgativas).

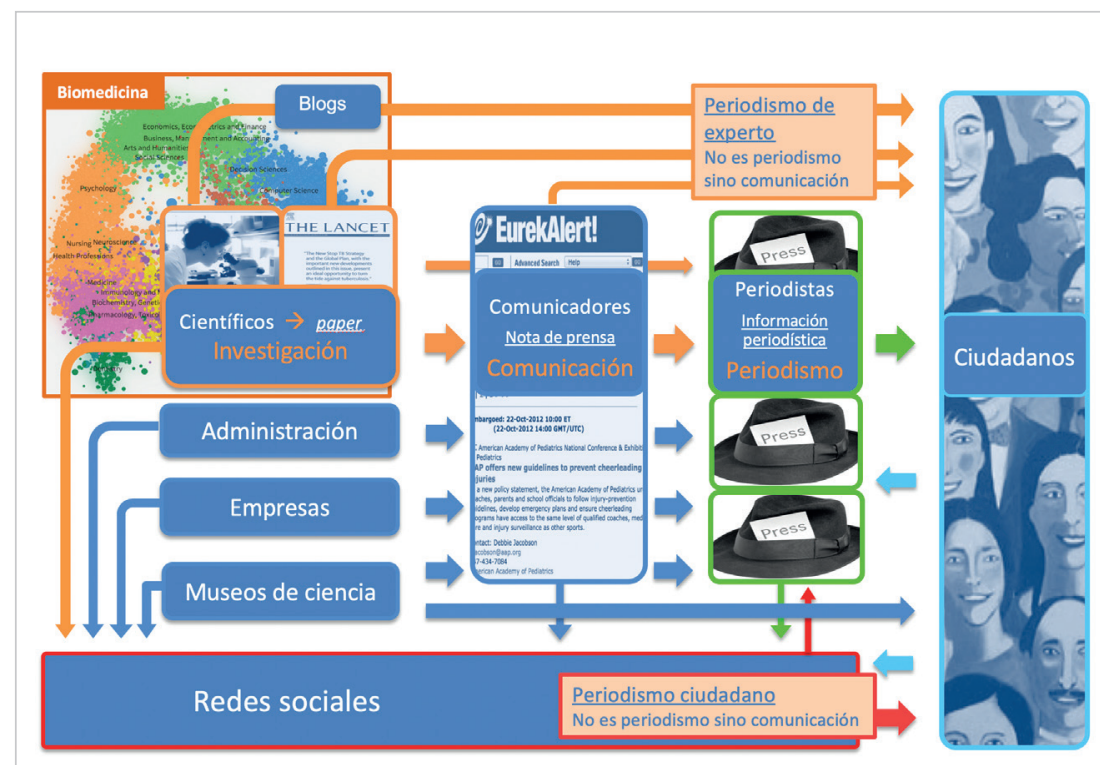

Figura 3. Agentes, flujos y actividades en el ecosistema de la información biomédica

\subsection{Agentes, actividades y flu- jos de información}

En la figura 3 se representa una imagen simplificada del ecosistema de la información biomédica. En él cabe distinguir tres actores principales: los productores de publicaciones sobre biomedicina (científicos, revistas, centros de investigación, etc.); los gabinetes de comunicación y comunicadores profesionales, que elaboran las notas de prensa y otros contenidos; y los periodistas y medios de comunicación, que producen noticias y otros materiales periodísticos. El cuarto agente sería el público, destinatario último de la información sobre biomedicina, que Las noticias se han convertido en el producto final de un sofisticado engranaje promocional en el que participan todos los actores del ecosistema de la información biomédica

ha dejado de ser un receptor pasivo.

Hasta la aparición de internet y las redes sociales, la comunicación seguía un flujo unidireccional a través de tres productos informativos principales: artículos científicos, notas de prensa y contenidos periodísticos, de los cuales sólo el último solía llegar al público. Este esquema tradicional, en el que el periodismo tenía prácticamente el monopolio de la intermediación entre la ciencia y el público, se ha ampliado con nuevos flujos y reflujos que permiten la interacción entre todos los agentes.

Los investigadores y demás productores de ciencia pueden comunicarse directamente con el público mediante blogs y webs. Asimismo, muchas notas de prensa están disponibles para el público, en las webs de las revistas e instituciones científicas y/o en los repositorios de comunicados como EurekAlert y AlphaGalileo (figuras 4 y 5 ). Las cada vez más numerosas revistas de acceso abierto también han favorecido este acercamiento de la biomedicina a la ciudadanía.

Estos nuevos flujos de información han abierto progresivamente el cerrado mundo de la biomedicina al público y han traído oportunidades de información y comunicación antes desconocidas o minoritarias, así como la posibilidad de

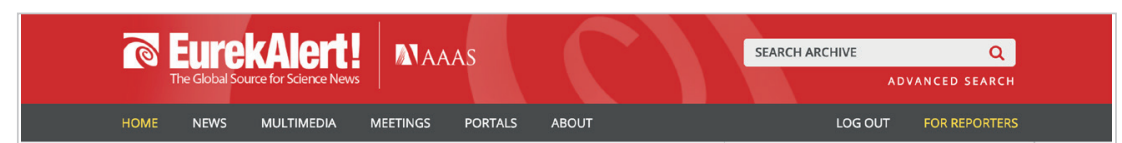

For Reporters
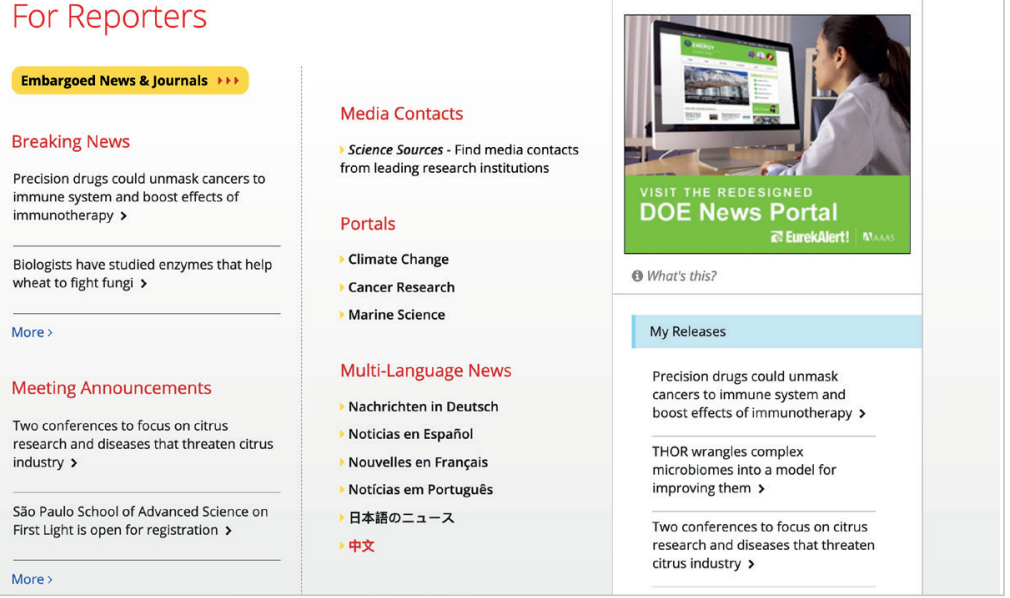

Figura 4. Repositorio de comunicados de prensa EurekAlert https://www.eurekalert.org 
participación del público en la llamada ciencia ciudadana. El periodismo también se ha visto beneficiado y a veces hasta suplantado en su tarea informativa, si bien hay que poner en cuestión la existencia tanto del periodismo ciudadano como del de experto, pues en ambas situaciones no se suelen cumplir los requisitos periodísticos de independencia, comprobación de la información y ausencia de conflictos de intereses.

Sin embargo, la cadena tradicional de tres eslabones formada por el artículo científico, la nota de prensa y el artículo periodístico sigue siendo el cauce principal de la información biomédica. La difusión de la mayoría de los resultados de la investigación se apoya todavía en la fortaleza de esta cadena. Con todo, existen recelos crecientes por parte de los periodistas, que se sienten instrumentalizados y no acaban de confiar en las redes sociales como fuente de información (Varona-Aramburu; Sánchez-Muñoz, 2016).

\subsection{Más comunicación y menos periodismo}

La crisis periodística iniciada a finales de la década de 2000 ha deparado un debilitamiento de las secciones de ciencia y salud en la mayoría de los medios de comunicación. El número de periodistas especializados ha disminuido y los de mayor experiencia han sido sustituidos por otros menos cualificados. En conjunto, en los medios tradicionales se informa menos sobre biomedicina y la información es más dependiente de los comunicados de prensa y la comunicación profesional. En contrapartida, han surgido algunos nuevos medios que ofrecen información abundante y de gran calidad sobre biomedicina, como ejemplifica el caso de Vox en Estados Unidos.

El auge de la comunicación, sin embargo, parece imparable. Prácticamente todos los centros de investigación y las universidades hacen difusión de sus investigaciones en internet y las redes sociales, aunque con una implicación desigual (López-Pérez; Olvera-Lobo, 2016). Además, algunos de los veteranos periodistas y buena parte de los jóvenes graduados acaban dedicándose a la comunicación, ya que esta actividad ofrece más oportunidades (Salaverría, 2016).

El resultado es que cada vez hay más comunicación y menos periodismo, y no es raro que la comunicación suplante al periodismo. Aunque faltan análisis rigurosos de este fenómeno, existen algunos indicios. La Asociación Española de Periodismo Científico, fundada en 1973, cambió su denominación tres décadas después por el de Asociación Española de Comunicación Científica, ampliando así sus objetivos y actividades para los socios; hoy, menos de la quinta parte de ellos trabaja como periodista. El crecimiento de un repositorio de comunicados de prensa como EurekAlert muestra también el vigor de la comunicación biomédica. Y, finalmente, la aparición de plataformas como Futurity (figura 6), cuyo contenido está formado por notas de prensa de numerosas universidades angloamericanas, evidencia la necesidad de dar salida a un excedente de comunicación que ya no encuentra cauces de difusión en los medios. La información de esta

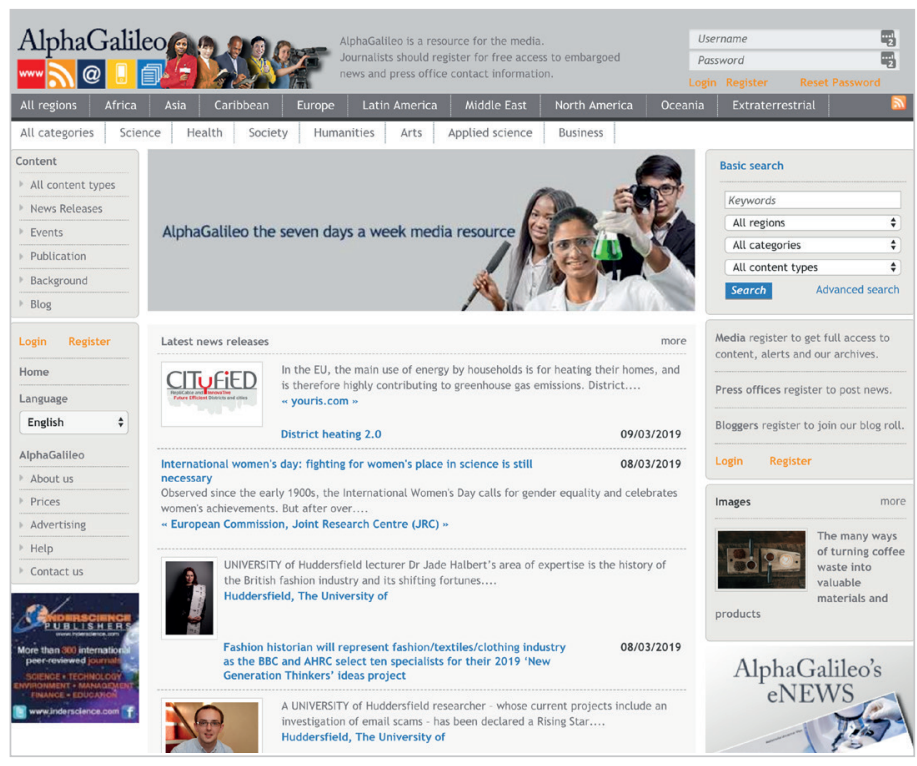

Figura 5. Repositorio de comunicados de prensa AlphaGalileo https://www.alphagalileo.org
El concepto que mejor condensa los problemas y disfunciones de la información biomédica es el de exageración (hype, en inglés)

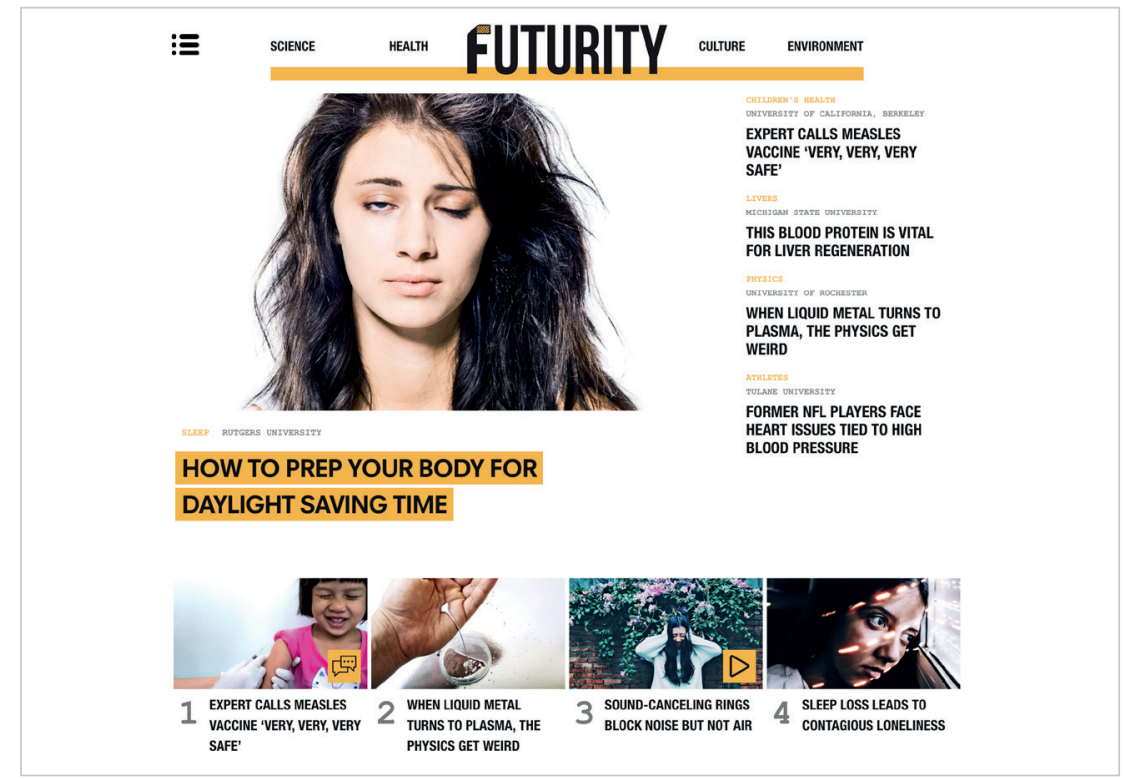

Figura 6. Futurity, web de información sobre la investigación en universidades https://www.futurity.org 
plataforma y otras similares no es periodismo (información desinteresada) sino comunicación (información interesada), del mismo modo que muchas informaciones periodísticas son poco más que notas de prensa.

\section{Problemas y disfunciones}

La información biomédica que llega al público, en especial a través de los medios de comunicación, tiene un gran valor para que la ciudadanía pueda tomar decisiones sobre su salud. Sin embargo, esta información es a menudo deficiente y, más que informar, puede desinformar y confundir, crear ansiedad y propiciar que el ciudadano tome decisiones equivocadas (Goldacre, 2007; Schwitzer, 2008). Numerosas investigaciones han constatado que las noticias médicas adolecen de sensacionalismo, incompletitud, imprecisión, intereses ocultos, sesgos varios, falta de rigor y otras deficiencias (Moynihan et al., 2000; Smith, 2005; Hochman et al., 2008; Reid; Malone, 2008; Lai; Lane, 2009; McGrath; Kapadia, 2009; Casino, 2015a). El problema de la desinformación se ha agravado paulatinamente con la recirculación de los mensajes en las redes sociales y su degradación en los sucesivos flujos y reflujos informativos.

El catálogo de problemas de la información que llega al público es extenso y complejo, pero resulta tan desatinado como débil intelectualmente responsabilizar en exclusiva al periodismo. Muchos periodistas carecen de la experiencia y las competencias necesarias para abordar una información compleja como la de biomedicina. Pero las noticias se han convertido en el producto final de un sofisticado engranaje promocional en el que participan todos los actores del ecosistema de la información biomédica, desde la industria farmacéutica y los centros de investigación hasta las revistas y sus gabinetes de comunicación, por lo que las responsabilidades están repartidas. La comercialización de la información biomédica se ha infiltrado en todo el proceso, en todos los niveles y actividades informativas. Richard Smith, el exdirector del British medical journal, lo expresó con rotundidad en el titular de un artículo de 2005 publicado en PLOS medicine:

"Las revistas médicas son una extensión de la división de marketing de las compañías farmacéuticas" (Smith, 2005).

Moynihan y Cassels (2006) fueron todavía más allá:

"Como casi todo lo que ocurre en la asistencia médica actualmente, las ideas que tenemos sobre la enfermedad han sido moldeadas bajo la sombra de los gigantes farmacéuticos mundiales".

\subsection{La exageración y sus máscaras}

El concepto que mejor condensa los problemas y disfunciones de la información biomédica es el de exageración (hype, en inglés). Afecta a los tres productos principales de la cadena informativa (artículos científicos, notas de prensa y artículos periodísticos) y se manifiesta en cada uno de ellos de forma diferente. En periodismo, la exageración se denomina también sensacionalismo y se puede definir como la brecha existente entre el mensaje científico y el mensaje periodístico. Por tanto, toda la información que no se ajuste a las pruebas científicas y los resultados de la investigación puede considerarse exagerada.

Pero la exageración también tiene sus máscaras y se manifiesta de otras formas. Por ejemplo, cuando se habla de salud humana basándose en resultados de estudios con animales, se alude a un aparatoso riesgo relativo sin mencionar el riesgo absoluto, se divulgan resultados preliminares de una investigación como si fueran definitivos y se difunden mensajes de salud que no están respaldados por evidencias científicas o se basan en pruebas de bajo o muy bajo grado de certeza sin decirlo expresamente.

Una variante sutil de la exageración es la incompletitud. Las informaciones sobre intervenciones médicas, en especial sobre tratamientos, son tan complejas y exigen considerar tantos aspectos que muy a menudo resultan sesgadas y de baja calidad por incompletas. Por debajo de las 300 palabras apenas hay noticias rigurosas sobre intervenciones médicas (Schwitzer, 2007), y este tipo de informaciones representan la cuarta parte de las noticias sobre biomedicina (Casino, 2015b).

Tradicionalmente se consideraba que la responsabilidad del sensacionalismo era prácticamente exclusiva del periodismo, pero se ha constatado que muchas de las exageraciones de las noticias están inducidas por las notas de prensa (Sumner et al., 2014). Esta conclusión se basa en el análisis de tres tipos de exageraciones en las noticias y los comunicados de prensa:

- inferencias en humanos de resultados con animales;

- afirmaciones causales a partir de simples correlaciones; y

- consejos de salud sin base en evidencias científicas.

Sus resultados indican que las exageraciones originadas en las noticias periodísticas no son frecuentes y normalmente se correlacionan con exageraciones en los comu-
Tradicionalmente se consideraba que la responsabilidad del sensacionalismo era exclusiva del periodismo, pero se ha constatado que muchas de las exageraciones de las noticias están inducidas por las notas de prensa 
nicados de prensa, confirmando que el periodismo biomédico cumple a menudo una función de correa de transmisión.

\subsection{Mala ciencia con impacto mediático}

La proliferación de la llamada mala ciencia o ciencia con deficiencias metodológicas es una de las principales amenazas a las que enfrenta la biomedicina. Aunque el concepto de mala ciencia, popularizado por Ben Goldacre en su columna Bad science del diario The guardian y en un libro homónimo (Goldacre, 2009), no está todavía bien definido, bajo esta denominación encaja la investigación hecha con datos falsificados o inventados, la injustificadamente sesgada y redundante, la irreproducible y, en general, la que produce resultados irrelevantes, falsos o inciertos como consecuencia de un mal diseño del estudio y una mala praxis.

Con su artículo Why most published research findings are false, loannidis (2005) alertó sobre la existencia de factores que aumentan la probabilidad de obtener resultados falsos, como la relajación en el diseño del estudio y la existencia de conflictos de interés. Esta mala ciencia es más frecuente en el campo de la biomedicina que en el de las ciencias naturales, y para caracterizarla ha sido también denominada irónicamente evidence-biased medicine (Jakovljević; Ostojić, 2016).

Sólo el 6\% de los 50.000 artículos anuales publicados en las 110 principales revistas de biomedicina se ha elaborado con una metodología correcta y ofrece resultados relevantes para la práctica clínica (Haynes, 2005). A esta abundancia de estudios irrelevantes y sesgados hay que sumar la desmesurada atención mediática que reciben, pues en la prensa predominan los estudios con resultados preliminares y menos contrastados, muchos de los cuales acaban demostrándose falsos (Dumas-Mallet et al., 2017; 2018). El caso de la cobertura periodística del síndrome de déficit de atención con hiperactividad es un ejemplo bien analizado de información periodísitica sobre resultados inciertos que acaban siendo falsos (Gonon et al., 2012).

\section{Retos en investigación, comunicación y periodismo}

El análisis y la comprensión de los problemas y disfunciones de la información biomédica es un reto global para todos los agentes. Los periodistas y comunicadores pueden hacer mucho por mejorar sus informaciones a partir del análisis crítico de su actividad, pero los académicos tienen una especial responsabilidad. Algunos de los investigadores que estudian el periodismo y la comunicación sobre biomedicina tienen experiencia como periodistas y/o comunicadores, lo cual facilita la comprensión de los problemas. Con todo, buena parte de los estudios sobre la información biomédica y su difusión son publicados por investigadores médicos en revistas médicas, de salud pública y de ciencia general, cada vez más abiertas a los temas de comunicación (Bermúdez-Tamayo et al., 2018), como prueba de lo importante que es para la salud la calidad de la información que reciben los ciudadanos.

\subsection{Analizar la calidad y sus componentes}

Comprender cuáles son los requisitos e ingredientes de la calidad en la información biomédica es un paso previo para mejorarla. El análisis de las noticias sobre medicamentos ha revelado que un gran número de ellas ofrecen información inadecuada e incompleta sobre los beneficios, los riesgos y los costes de los medicamentos, así como sobre la financiación de los estudios y los conflictos de interés, entre otros factores (Moynihan et al., 2000; Cassels et al., 2003; Hochman et al., 2008). Diversas iniciativas en Australia, Canadá y Estados Unidos han culminado con el consenso de una decena de criterios para evaluar la calidad de las noticias sobre intervenciones médicas (tratamientos farmacológicos, tratamientos quirúrgicos, pautas dietéticas y pruebas diagnósticas, entre otras). La evaluación más completa es la realizada por HealthNewsReview (figura 7), que desde 2004 a 2018 ha analizado la calidad de más

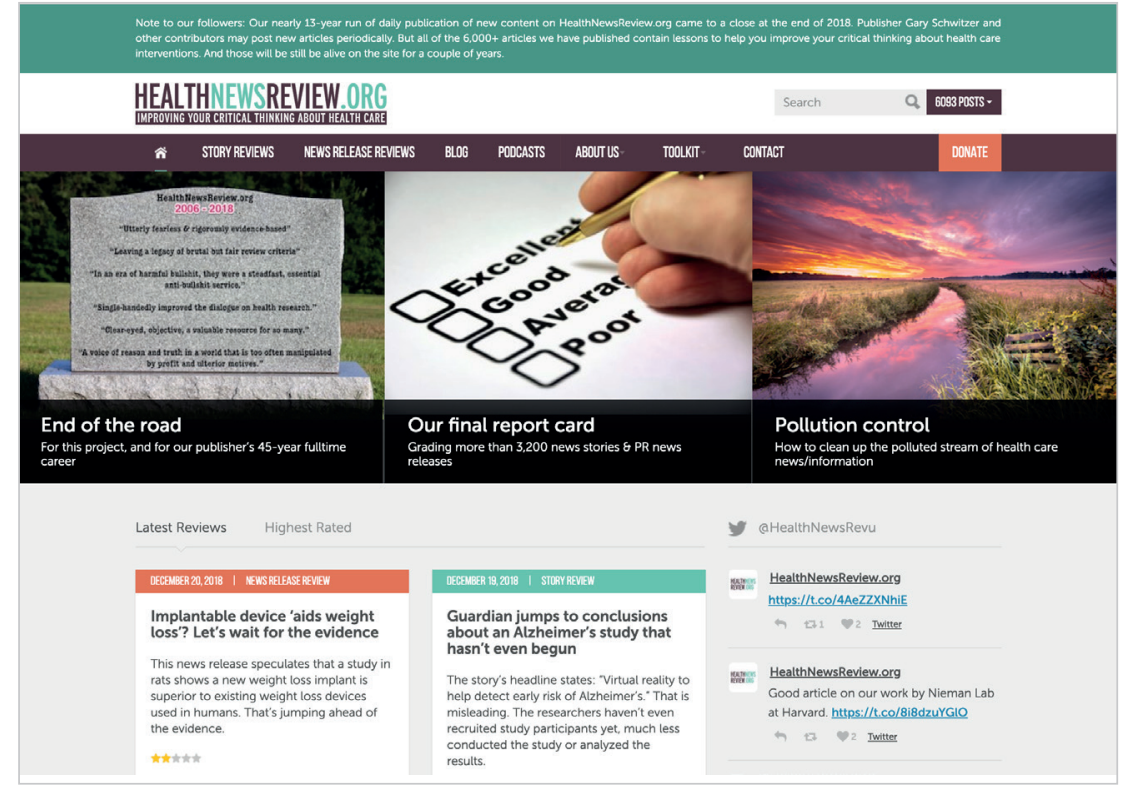

Figura 7. Proyecto HealthNewsReview de evaluación de la calidad de las noticias sobre intervenciones médicas

https://www.healthnewsreview.org 
de 2.600 artículos periodísticos y más de 600 comunicados de prensa en Estados Unidos, detectando una gran mediocridad pero también una cierta mejoría (Schwitzer, 2008; Walsh-Childers et al., 2018).

Para medir la calidad de las noticias de biomedicina existen diversas herramientas, desde el primer índice de calidad de Oxman et al. (1993) hasta las más recientes de Robinson et al. (2013) y Zeraatkar et al. (2017). Pero todas tienen sus carencias y debilidades (Bosch et al., 2018), probablemente porque han sido elaboradas desde la biomedicina sin el concurso de expertos en periodismo. La disponibilidad de una mejor escala de calidad para evaluar las noticias y las notas de prensa facilitaría la investigación y, probablemente, una mejora en la calidad de la información.

\subsection{Conocer el valor noticioso de la biomedicina y su impacto mediático}

La información sobre biomedicina que difunden los medios de comunicación no es exactamente la que más le interesa al público, ni tampoco la más relevante desde el punto de vista científico o educativo. Una cosa es el valor noticioso de la biomedicina (newsworthiness, en inglés) y otra lo que finalmente se convierte en noticia. Las noticias son un producto informativo que se puede comprar y vender, mientras que el valor noticioso, interés periodístico o noticiabilidad, es un juicio psicológico. Este interés periodístico resulta ser muy similar para todo tipo de países y personas, sin importar su condición socioeconómica y su profesión (Shoemaker, 2006).

El valor noticioso de la información es un factor importante pero no es un buen predictor de lo que acaba convirtiéndose en noticia. La publicación de una noticia de biomedicina obedece a una serie de factores, entre los que se cuentan la existencia de una nota de prensa asociada (De-Semir, 1998; Schwartz et al., 2012; Yavchitz et al., 2012; Casino, 2015; Sumner et al., 2016), la relevancia científica de la información (Repiso; Chaparro-Domínguez, 2018) y un factor nacional que prima la difusión de la investigación de un país en los medios nacionales (Casino; Rius; Cobo, 2017). Pero la lista completa de factores asociados con la cobertura periodística y la importancia relativa de cada uno, en particular el peso de los intereses económicos y profesionales asociados a la información, está lejos de ser comprendida. En este sentido, el análisis de las citas periodísticas puede ser una buena herramienta para cuantificar el impacto mediático de la información biomédica y analizar su correlación con algunas variables (Casino, 2018).

\subsection{Combatir la exageración y sus consecuencias}

La principal consecuencia negativa de la exageración es que genera falsas expectativas y alarmas infundadas entre los ciudadanos, y puede favorecer, por tanto, la toma de decisiones equivocadas sobre la propia salud. El problema es complejo, pues hay numerosos factores que favorecen el sensacionalismo, desde la competencia entre los medios de comunicación a la necesidad de los científicos de dar publicidad a sus investigaciones para consolidar su prestigio y captar financiación (Schwartz; Woloshin, 2003). Como han destacado Ransohoff y Ransohoff (2001), la exageración hunde sus raíces en la complicidad entre científicos y periodistas, pues existe un beneficio mutuo que alimenta el fenómeno: los medios consiguen audiencia y los científicos, visibilidad.

Esta visibilidad de la investigación tiene beneficios tangibles para los autores. Desde finales del siglo XX se ha constatado que la cobertura periodística se asocia con más citas en artículos académicos (Phillips et al., 1991; Kiernan, 2003; Fanelli, 2013). Más recientemente también se ha asociado con una mayor descarga de esos ar-

La información sobre biomedicina que difunden los medios de comunicación no es la que más le interesa al público, ni tampoco la más relevante desde el punto de vista científico o educativo

tículos en internet (Mathelus; Pittman; Yablonski-Crepeau, 2012). Las notas de prensa son, en este sentido, la principal herramienta para conseguir impacto en los medios y esos beneficios asociados. Como han constatado numerosos estudios, la difusión de la investigación biomédica en la prensa se correlaciona fuertemente (entre el $80 \%$ y el 100\%, según los estudios) con la existencia de una nota de prensa relacionada.

Aunque los comunicadores que elaboran las notas de prensa y los periodistas que se hacen eco de ellas pueden hacer mucho por mejorar la comprobación de datos (fact checking) (Ufarte-Ruiz; Peralta-García; Murcia-Verdú, 2018) y la calidad de sus respectivas informaciones, los importantes intereses económicos y profesionales que hay en juego dificultan la prevención de la exageración. Como señalan Schwartz y Woloshin (2003), más realista que prevenir la exageración es tratar de remediar sus efectos enseñando a los ciudadanos a desarrollar un escepticismo saludable frente a la información. Este es un reto importante que incumbe a todos.

\section{Perspectivas: cambios de tempo y mayor colaboración}

Los cambios en el ecosistema de la información biomédica han traído nuevas posibilidades de ofrecer buena y mala información. Nunca como ahora ha habido tantas oportunidades para la investigación excelente, la comunicación innovadora y el periodismo independiente y riguroso. Pero, simultáneamente, nunca ha habido tanta mala ciencia, comunicación deficiente y periodismo ajeno al interés público. Faltan datos para hacer un balance y anticipar qué tendencia 
es más fuerte, pero se observa un fenómeno curioso: mientras el periodismo biomédico se hace más científico, la biomedicina se hace más mediática. En cierta manera, las prisas están cambiando de lado: mientras el periodismo está descubriendo las ventajas del sosiego y el trabajo concienzudo, hay señales de precipitación y superficialidad en la investigación.
Las noticias son un producto informativo que se puede comprar y vender, mientras que el valor noticioso, interés periodístico o noticiabilidad, es un juicio psicológico

Las noticias de biomedicina no suelen necesitar una difusión inmediata. Son noticias blandas o soft, por oposición a las duras o hard, que no pueden esperar porque dejarían de ser noticia, como es el caso de la mayoría de las de política, deportes o sucesos (Shoemaker, 2006). No hay, por tanto, razones para las prisas y la precipitación, si el precio que hay que pagar es la desinformación (Woloshin; Schwartz, 2006). Esto es algo que está descubriendo una parte menor pero significativa del periodismo, que empieza a desdeñar las investigaciones preliminares, como las que se presentan en congresos, y está aprendiendo a integrar la incertidumbre en la información.

En la prensa hay una clara preferencia por los estudios de menor grado de certeza. Los periódicos informan más de los estudios observacionales (46\%) que de los ensayos clínicos (21\%) y las revisiones sistemáticas (3\%); las demás noticias sobre biomedicina se refieren a estudios con animales (17\%) y opiniones de expertos (14\%) (Lai; Lane, 2009). Pero en el periodismo se empieza a observar una tendencia hacia la información más completa, en la que los nuevos estudios se interpretan a la luz del conjunto de la investigación y se considera el grado de certeza de los resultados. Los análisis de la calidad de las noticias médicas muestran una mejoría desde 2010 (Walsh-Childers; Braddock; Rabaza; Schwitzer, 2018).

El periodismo empieza también a integrar algunos elementos propios de la ciencia, como son el análisis de datos y variables, la transparencia, la reproducibilidad y el trabajo en equipo. Nuevas fuentes de financiación a través de instituciones y fundaciones, con convocatorias competitivas y largos plazos de realización, están dando lugar a nuevos trabajos periodísticos que combinan la metodología científica y el reporterismo, como los de la Fundación Civio.

https://civio.es

La mala ciencia está inundando el ecosistema de publicaciones biomédicas de mala calidad que aportan ruido y confusión. A la presión y las prisas por publicar, reconocidas por los propios investigadores (Belluz; Plumer; Resnick, 2016), hay que añadir el creciente interés de que la biomedicina sea mediática y aporte visibilidad a las investigaciones. Aunque la percepción pública de la medicina y la investigación biomédica es muy favorable, hay signos de deterioro que podrían dañar su credibilidad. Hay además una grave crisis de reproducibilidad en la ciencia, que atenta contra este pilar esencial del conocimiento científico y que afecta más a la biomedicina que a otras áreas como la física o la química (Baker, 2016). Esta crisis todavía no ha trascendido a la sociedad, pero preocupa a un número creciente de investigadores. Algunos de ellos ya han propuesto medidas para mejorar la reproducibilidad y la credibilidad de la biomedicina y la ciencia en general (Munafò et al., 2017).

Mientras la ciencia hace autocrítica para corregir sus deficiencias y el periodismo explora nuevas vías para ganar credibilidad, la comunicación profesional sigue inundando los medios y las redes de mensajes interesados. Como reacción, algunas iniciativas tratan de mejorar la calidad de los comunicados de prensa, las competencias divulgativas de los investigadores y las científicas de los periodistas. Pero todavía parecen lejanas la consideración de la divulgación como un mérito en la carrera profesional del científico y la exigencia de alguna acreditación para los periodistas que informan cobre biomedicina, dos propuestas que quizá podrían mejorar la calidad de la información.

Un aspecto destacable es la creciente colaboración entre los distintos agentes del ecosistema de la información biomédica en proyectos para ayudar al público general a interpretar los mensajes de salud y a desarrollar un pensamiento crítico, como por ejemplo los vinculados a la Colaboración Cochrane (figura 8). En estos tiempos de verdades relativas, medias verdades y noticias falsas quizá sea esta la mejor alternativa.

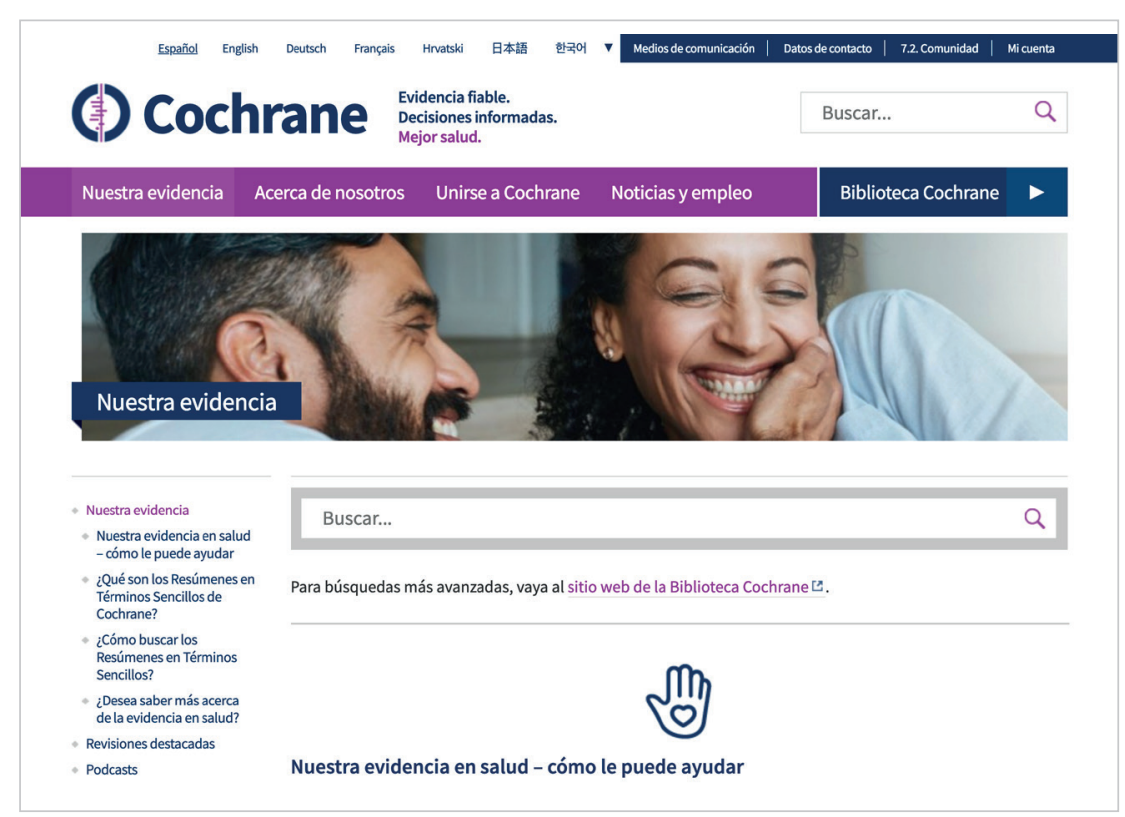

Figura 8. Colaboración Cochrane

https://www.cochranelibrary.com/es 


\section{Referencias}

Baker, Monya (2016). "1,500 scientists lift the lid on reproducibility". Nature, v. 533, n. 7604, pp. 452-454. https://doi.org/10.1038/533452a

Bauer, Martin (1998). "The medicalization of science news - from the "rocket-scalpel" to the 'gene-meteorite' complex". Social science information, v. 37, n. 4, pp. 731-751.

https://doi.org/0803973233

Belluz, Julia; Plumer, Brad; Resnick, Brian (2016). "The 7 biggest problems facing science, according to 270 scientists". Vox, Sept. $7^{\text {th }}$.

http://www.vox.com/2016/7/14/12016710/science-challeges-research-funding-peer-review-process

Bermúdez-Tamayo, Clara; Negrín-Hernández, Miguel; Alguacil, Juan; Briones-Vozmediano, Erica; Cantarero, David; Carrasco-Portiño, Mercedes; Casino, Gonzalo; Castro-Sánchez, Enrique; García-Calvente, Mar; González-Zapata, Laura-Inés; Epstein, David; Hernan, Mariano; Linares, Cristina; Posenato-García, Leila; Ruiz-Cantero, María-Teresa; Segura, Andreu; Zunzunegui, María-Victoria; Sarria, Antonio; Peiro, Rosana; Álvarez-Dardet, Carlos (2018). "Gaceta Sanitaria en 2017. Mejorando la calidad de nuestra revista". Gaceta sanitaria, v. 32, n. 2, pp. 117-120.

https://doi.org/10.1016/j.gaceta.2018.02.002

Bosch, Fèlix; Escalas, Clàudia; Forteza, Ainoa; Serés, Elisabet; Casino, Gonzalo (2018). “Lista de comprobación para mejorar la información sobre fármacos en la prensa: la importancia de informar sobre las fases y la incertidumbre de la investigación". Revista española de comunicación en salud, v. 9, n. 2, pp. 203-214.

https://doi.org/10.20318/recs.2018.4498

Camí, Jordi; Méndez-Vásquez, Raúl; Suñén-Pinyol, Eduard (2008). “Evolución de la productividad científica de España en biomedicina (1981-2006)". Redes, v. 10, pp. 24-29.

Casino, Gonzalo (2015a). “Concisión frente a completitud en las noticias médicas. Análisis de los breves de biomedicina en el diario El País". Panacea, v. 16, n. 42, pp. 184-189.

http://www.tremedica.org/panacea/IndiceGeneral/n42_tribuna-GCasino2.pdf

Casino, Gonzalo (2015b). "Información de las revistas de biomedicina mediada por comunicados de prensa. El caso del diario El País". Panacea, v. 16, n. 42, pp. 151-157.

http://www.tremedica.org/panacea/IndiceGeneral/n42_tribuna-GCasino.pdf

Casino, Gonzalo (2018). "Cita periodística: impacto de las revistas y los artículos científicos en la prensa generalista". El profesional de la información, v. 27, n. 3, p. 692.

https://doi.org/10.3145/epi.2018.may.22

Casino, Gonzalo; Rius, Roser; Cobo, Erik (2017). "National citation patterns of NEJM, The Lancet, JAMA and The BMJ in the lay press: a quantitative content analysis". BMJ Open, v. 7, n. 11, p. e018705.

https://doi.org/10.1136/bmjopen-2017-018705

Cassels, Alan; Hughes, Merrilee A.; Cole, Carol; Mintzes, Barbara; Lexchin, Joel; McCormack, James P. (2003). “Drugs in the news: an analysis of Canadian newspaper coverage of new prescription drugs". CMAJ, v. 168, n. 9, pp. 1133-1137. http://www.cmaj.ca/content/168/9/1133.short

De-Semir, Vladimir; Ribas, Cristina; Revuelta, Gemma (1998). "Press releases of science journal articles and subsequent newspaper stories on the same topic". Jama, v. 280, n. 3, pp. 294-295.

https://doi.org/10.1001/jama.280.3.294

Dumas-Mallet, Estelle; Smith, Andy; Boraud, Thomas; Gonon, François (2017). "Poor replication validity of biomedical association studies reported by newspapers". Plos one, v. 12, n. 2, p. e0172650.

https://doi.org/10.1371/journal.pone.0172650

Dumas-Mallet, Estelle; Smith, Andy; Boraud, Thomas; Gonon, François (2018). "Scientific uncertainty in the press: how newspapers describe initial biomedical findings". Science communication, v. 40, n. 1, pp. 124-141.

https://doi.org/10.1177/1075547017752166

Fanelli, Daniele (2013). "Any publicity is better than none: Newspaper coverage increases citations, in the UK more than in Italy". Scientometrics, v. 95, n. 3, pp. 1167-1177.

https://doi.org/10.1007/s11192-012-0925-0

Fecyt (2018). "IX encuesta de percepción social de la ciencia y la tecnología en España. Informe de resultados". https://icono.fecyt.es/informes-y-publicaciones/percepcion-social-de-la-ciencia-y-la-tecnologia-en-espana

Goldacre, Ben (2007). "How doctors can get behind the headlines". British medical journal, v. 334, n. 7594 , p. 613. https://doi.org/10.1136/bmj.39160.566285.47 
Goldacre, Ben (2009). Bad science. London: Harper Perennial. ISBN: 9780007284870

Gonon, François; Konsman, Jan-Pieter; Cohen, David; Boraud, Thomas (2012). "Why most biomedical findings echoed by newspapers turn out to be false: The case of attention deficit hyperactivity disorder". Plos one, v. 7, n. 9, p. e44275. https://doi.org/10.1371/journal.pone.0044275

Haynes, R. Brian (2005). "bmjupdates+, a new free service for evidence-based clinical practice". Evidence-based nursing, v. 8, n. 2 , p. 39.

https://doi.org/10.1136/EBN.8.2.39

Hochman, Michael; Hochman, Steven; Bor, David; McCormick, Danny (2008). "News media coverage of medication research: reporting pharmaceutical company funding and use of generic medication names". Jama, v. 300, n. 13, pp. 1544-1550.

https://doi.org/10.1001/jama.300.13.1544

loannidis, John P. A. (2005). "Why most published research findings are false". Plos medicine, v. 2, n. 8, e124. https://doi.org/10.1371/journal.pmed.0020124

Jakovljević, Miro; Ostojić, Ljerka (2016). "Science and pseudoscience in medicine: evidence-based vs. evidence-biased medicine". Psychiatria danubina, v. 28, n. Suppl-2, pp. 186-190.

http://www.psychiatria-danubina.com/UserDocs/mages/pdf/dnb_vol28_sup2/dnb_vol28_sup2_2.pdf

Kiernan, Vincent (2003). “Diffusion of news about research". Science communication, v. 25, n. 1, pp. 3-13.

https://doi.org/10.1177/1075547003255297

Lai, William-Yuk-Yeu; Lane, Trevor (2009). "Characteristics of medical research news reported on front pages of newspapers". Plos one, v. 4, n. 7, e6103.

https://doi.org/10.1371/journal.pone.0006103

López-Pérez, Lourdes; Olvera-Lobo, María-Dolores (2016). “Comunicación pública de la ciencia a través de la web 2.0. El caso de los centros de investigación y universidades públicas de España”. El profesional de la información, v. 25, n. 3, p. 441.

https://doi.org/10.3145/epi.2016.may.14

Mathelus, Sharon; Pittman, Ginny; Yablonski-Crepeau, Jill (2012). "Promotion of research articles to the lay press: a summary of a three-year project". Learned publishing, v. 25, n. 3, pp. 207-212.

https://doi.org/10.1087/20120307

McGrath, Brent M.; Kapadia, Ronak K. (2009). "Is the medium distorting the message? How the news media communicates advances in medical research to the public". Dalhousie medical journal, v. 36, n. 1, pp. 11-17.

https://doi.org/10.15273/dmj.Vol36No1.3871

Moynihan, Ray; Bero, Lisa; Ross-Degnan, Dennis; Henry, David; Lee, Kirby; Watkins, Judy; Mah, Connie; Soumerai, Stephen B. (2000). "Coverage by the news media of the benefits and risks of medications". New England journal of medicine, v. 342, n. 22, pp. 1645-1650.

https://doi.org/10.1056/NEJM200006013422206

Moynihan, Ray; Cassels, Alan (2006). Medicamentos que nos enferman e industrias farmacéuticas que nos convierten en pacientes, Barcelona: Terapias Verdes, ISBN 978-84-96194-88-4

Munafò, Marcus R.; Nosek, Brian A.; Bishop, Dorothy V. M.; Button, Katherine S.; Chambers, Christopher D.; Percie du Sert, Nathalie; Simonsohn, Uri; Wagenmakers, Eric-Jan; Ware, Jennifer J.; Ioannidis, John P. A. (2017). "A manifesto for reproducible science". Nature human behaviour, v. 1, n. 1, p. 0021.

https://doi.org/10.1038/s41562-016-0021

Oxman, Andrew D.; Guyatt, Gordon H.; Cook, Deborah J.; Jaeschke, Roman; Heddle, Nancy; Keller, Jana (1993). “An index of scientific quality for health reports in the lay press". Journal of clinical epidemiology, v. 46, n. 9, pp. 987-1001.

Phillips, David P.; Kanter, Elliot J.; Bednarczyk, Bridget; Tastad, Patricia L. (1991). "Importance of the lay press in the transmission of medical knowledge to the scientific community". New England journal of medicine, v. 325, n. 16, pp. 1180-1183.

https://doi.org/10.1056/NEJM199110173251620

Reid, Adam J.; Malone, Paul S. C. (2008). "Plastic surgery in the press". Journal of plastic, reconstructive and aesthetic surgery, v. 61, n. 8, pp. 866-869.

https://doi.org/10.1016/j.bjps.2008.06.012

Repiso, Rafael; Chaparro-Domínguez, María-Ángeles (2018). “Universidades españolas en la prensa extranjera. Análisis de su cobertura periodística”. El profesional de la información, v. 27, n. 1, pp. 86-94.

https://doi.org/10.3145/epi.2018.ene.08 
Robinson, Ailie; Coutinho, Anastasia J.; Bryden, A. N.; McKee, Martin (2013). "Analysis of health stories in daily newspapers in the UK". Public health, v. 127, n. 1, pp. 39-45.

https://doi.org/10.1016/j.puhe.2012.10.001

Salaverría, Ramón (2016). "Redefinir al comunicador". El profesional de la información, v. 25, n. 2, pp. 163-167. https://doi.org/10.3145/epi.2016.mar.02

Schwartz, Lisa M.; Woloshin, Steven (2003). "On the prevention and treatment of exaggeration". Journal of general internal medicine, v. 18, n. 2, pp. 153-154.

https://doi.org/10.1046/j.1525-1497.2003.21216.x

Schwartz, Lisa M.; Woloshin, Steven; Andrews, Alice; Stukel, Therese A. (2012). "Influence of medical journal press releases on the quality of associated newspaper coverage: retrospective cohort study". British medical journal, v. 344, d8164.

https://doi.org/10.1136/bmj.d8164

Schwitzer, Gary (2007). "Too brief to matter - Part two: The benefits/harms of briefs and digests". Health news review, Sept. $14^{\text {th }}$.

https://www.healthnewsreview.org/2007/09/oo-brief-to-matter-part-two-the-benefitsharms-of-briefs-and-digests/

Schwitzer, Gary (2008). “How do US journalists cover treatments, tests, products, and procedures? An evaluation of 500 stories". Plos medicine, v. 5, n. 5, pp. 0700-0704.

https://doi.org/10.1371/journal.pmed.0050095

Shoemaker, Pamela J. (2006). "News and newsworthiness: a commentary". Communications, v. 31, n. 1, pp. 105-111. https://doi.org/10.1515/COMMUN.2006.007

Smith, Richard (2005). "Medical journals are an extension of the marketing arm of pharmaceutical companies". Plos medicine, v. 2, n. 5, pp. 0364-0366.

https://doi.org/10.1371/journal.pmed.0020138

Sumner, Petroc; Vivian-Griffiths, Solveiga; Boivin, Jacky; Williams, Andrew; Bott, Lewis; Adams, Rachel; Venetis, Christos A.; Whelan, Leanne; Hughes, Bethan; Chambers, Christopher D. (2016). "Exaggerations and caveats in press releases and health-related science news". Plos one, v. 11, n. 12, e0168217.

https://doi.org/10.1371/journal.pone.0168217

Sumner, Petroc; Vivian-Griffiths, Solveiga; Boivin, Jacky; Williams, Andy; Venetis, Christos A.; Davies, Aimée; Ogden, Jack; Whelan, Leanne; Hughes, Bethan; Dalton, Bethan; Boy, Fred; Chambers, Christopher D. (2014). "The association between exaggeration in health related science news and academic press releases: retrospective observational study". British medical journal, v. 349, g7015.

https://doi.org/10.1136/bmj.g7015

Ufarte-Ruiz, María-José; Peralta-García, Lidia; Murcia-Verdú, Francisco-José (2018). “Fact checking: un nuevo desafío del periodismo". El profesional de la información, v. 27, n. 4, p. 733-741.

https://doi.org/10.3145/epi.2018.jul.02

Varona-Aramburu, David; Sánchez-Muñoz, Gema (2016). “Las redes sociales como fuentes de información periodística: motivos para la desconfianza entre los periodistas españoles”. El profesional de la información, v. 25, n. 5, p. $795-802$. https://doi.org/10.3145/epi.2016.sep.10

Walsh-Childers, Kim; Braddock, Jennifer; Rabaza, Cristina; Schwitzer, Gary (2018). "One step forward, one step back: changes in news coverage of medical interventions". Health communication, v. 33, n. 2, pp. 174-187.

https://doi.org/10.1080/10410236.2016.1250706

Woloshin, Steven; Schwartz, Lisa M. (2006). "What's the rush? The dissemination and adoption of preliminary research results". Journal of the National Cancer Institute, v. 98, n. 6, pp. 372-373.

https://doi.org/10.1093/jnci/djj115

Yavchitz, Amélie; Boutron, Isabelle; Bafeta, Aida; Marroun, Ibrahim; Charles, Pierre; Mantz, Jean; Ravaud, Philippe (2012). "Misrepresentation of randomized controlled trials in press releases and news coverage: a cohort study". Plos medicine, v. 9, n. 9, e1001308.

https://doi.org/10.1371/journal.pmed.1001308

Zeraatkar, Dena; Obeda, Michael; Ginsberg, Jeffrey S.; Hirsh, Jack (2017). "The development and validation of an instrument to measure the quality of health research reports in the lay media". BMC public health, v. 17, n. 1, pp. 1-10. https://doi.org/10.1186/s12889-017-4259-y 